\title{
Multiple myeloma and COVID-19
}

\author{
Binod Dhakal ${ }^{1} \cdot$ Anita $\mathrm{D}^{\prime}$ Souza ${ }^{1} \cdot$ Saurabh Chhabra ${ }^{1} \cdot$ Parameswaran Hari $^{1}$
}

Received: 30 April 2020 / Revised: 7 May 2020 / Accepted: 13 May 2020 / Published online: 1 June 2020

C) Springer Nature Limited 2020

He et al. reported the outcomes of CVOID-19 in patients with hematological cancers in this Journal (April issue) [1]. They reported that hospitalized patients with hematological cancers have similar infection rates but more severe disease and case fatality rates. Few reports prior to this, reported the outcomes of COVID-19 patients with cancer [2-4]; however, none of them included patients with hematological malignancies like multiple myeloma (MM) [5]. Patients with $\mathrm{MM}$ are uniquely susceptible to viral and bacterial illnesses with an $~ 7-10$-fold higher risk of infection [6]. In addition, MM and its treatments lead to humoral immune deficiency, hypogammaglobulinemia and impaired B-cell responses to SARS-CoV-2. MM also places patients at higher risk of secondary infections. Since only three MM patients had COVID-19 in their series [1], the exact severity and outcomes of COVID-19 in the context of MM is not really known. We therefore conducted an urgent retrospective case study of $\mathrm{MM}$ patients diagnosed with COVID-19 at our institution.

We reviewed the medical records, demographics and outcomes of MM patients with laboratory confirmed COVID-19 from March 15, 2020 to April 18, 2020. The study was approved by the Institutional Review Board from Medical College of Wisconsin.

A total of seven MM patients in our practice were diagnosed with COVID-19 during the time period. Table 1 gives the baseline demographics of these patients. Ages ranged from 48 to 82 years, but $>70 \%$ patients were 65 years and older and $70 \%$ were females. Three $(43 \%)$ patients were African- Americans, and hypertension was the most common co-morbidity. Majority were on earlier lines of treatment with one receiving induction treatment, while two were on lenalidomide maintenance after autologous hematopoietic cell transplantation. Both of them were instructed to hold off

Binod Dhakal

bdhakal@mcw.edu

1 Division of Hematology/Oncology, Medical College of Wisconsin, Wauwatosa, WI, USA maintenance therapy. One patient had smoldering myeloma and never been on treatment. None of the patients had history of recent travel; or a cancer clinic visit in the 2-week interval prior to the development of symptoms strongly suggesting the community acquisition. Only one patient reported exposure to known COVID-19 case. Two patients had documented hypogammaglobulinemia at latest measurement (Fig. 1), both of whom had exposure to daratumumab.

Consistent with general population, the most common presenting symptoms were high fever, dry cough, and progressive shortness of breath. In one patient (\#6), GI symptoms (nausea, vomiting, and diarrhea) preceded dry cough and shortness of breath. All patients were hospitalized except one (\#2) who was younger and had milder symptoms. Her symptoms completely resolved after supportive care and self-isolation at home. Patient \#3 presented to emergency room a week prior with high fever and cough and was discharged home on azithromycin and amoxicillin after getting swab for CVOID-19. Two days later, she complained of sudden onset of difficulty in breathing leading to cardiac arrest leading to immediate death. Her swab was positive for COVID-19. Most of the hospitalized patients were treated with hydroxychloroquine and/or azithromycin except for one (\#4) who couldn't be treated due to long QT interval. This patient also had superimposed pneumonia due to streptococcus pneumoniae that was treated with antibiotics resulting in symptomatic resolution and subsequently discharged home. Majority of the hospitalized patients were monitored in the intensive care unit, and one needed mechanical ventilation. C-reactive protein (CRP) level was available in 4 patients and reached its peak at $20.2 \mathrm{mg} / \mathrm{dL}$ in patients \# 1 and \#4, and $15.3 \mathrm{mg} / \mathrm{dl}$ in \#7, while it remained within normal limits for patient \#5. Patient \# 1 developed progressive hypoxemic respiratory failure during the hospital stay and passed away after 5 days in the ICU as she declined intubation. None of the patients were treated with tocilizumab. Four of seven (57\%) patients died. The cause of death in three patients was progressive hypoxemic respiratory failure, while one patient had cardiac arrest at home. Patient \# 5 was discharged to nursing facility 


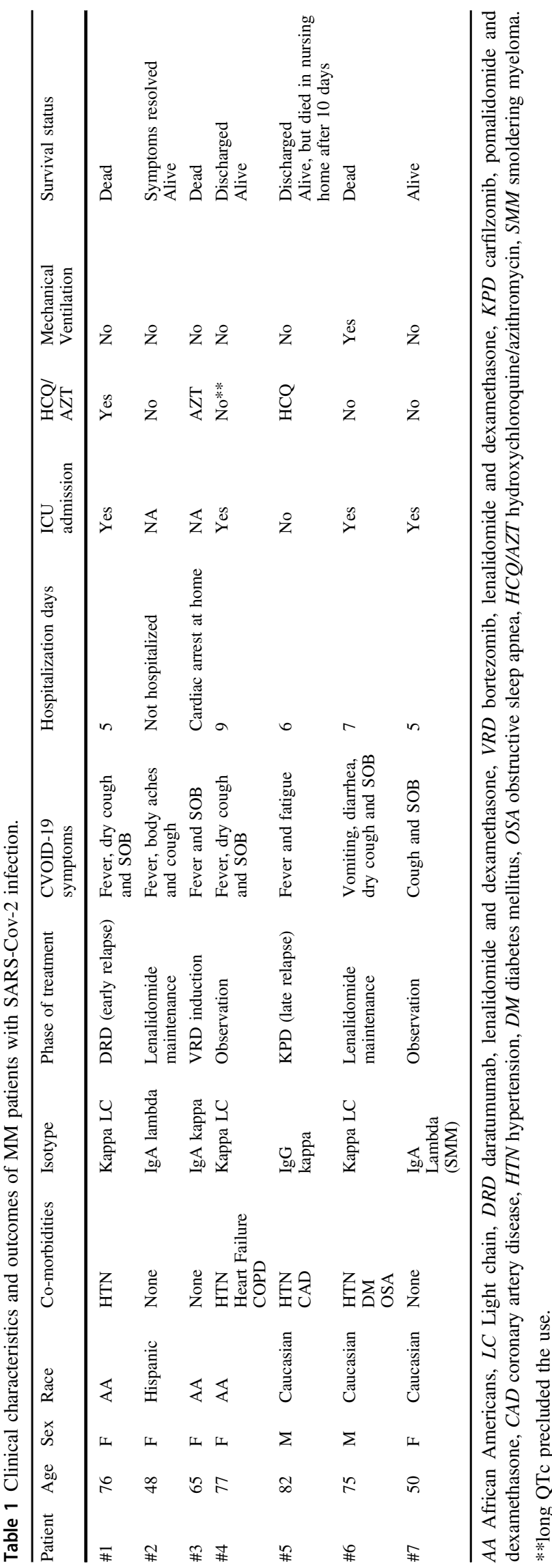

$\operatorname{lgG}(\mathrm{mg} / \mathrm{dl})$

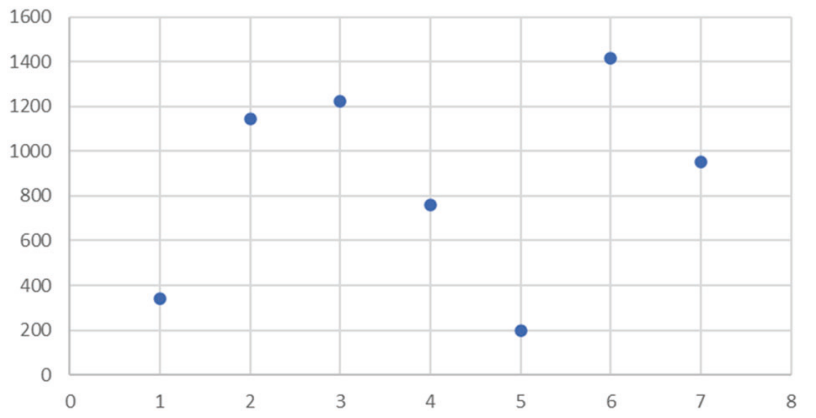

Fig. 1 Immunoglobulin G (IgG) $\mathrm{mg} / \mathrm{dl}$ level for each individual patient at the time of COVID-19 diagnosis. Total immunoglobulin G levels in patients in the latest measurement.

after clinical improvement, but unfortunately developed fever and respiratory distress after 5 days and transitioned to comfort care after discussion with the family.

Our case series specific for MM patients demonstrated the severity of COVID-19 as shown by He et al. [1]. A single case of MM patient with COVID-19 that was successfully treated with humanized IL-6 receptor antibody tocilizumab was recently reported [7]. A crucial cytokine involved in the pathogenesis of MM, IL-6, has also been implicated in the cytokine storm associated with severe COVID-19. In this series, none of the patients received tocilizumab. This could be due to the fact that CRP level, a surrogate marker of IL-6 driven inflammation, was elevated in three patients, two of which improved with supportive measures while the other declined further care upon respiratory decompensation. It will be important to see the effect of IL-6 receptor antibody in myeloma patients in the future larger studies. Despite the limitations of low number of patients, and retrospective nature of evidence, our data highlights some important observations. The mortality rate of 57\% overall and 4/5 hospitalized patients needing intensive care admission underscores the severity of this disease in the context of MM. Notably, the severity of COVID-19 did not seem to be related to MM status as patients on initial induction; early relapse and late relapse were all represented in our series.

This series originates from Wisconsin which has thus far had only moderate community (4000 cases with 779 / million cases) spread of COVID-19. The loss of four patients from among 2000 patients in our MM program is indicative of the severity of this disease in MM. While awaiting larger studies re: asymptomatic infection and serologic prevalence, we should recognize the serious consequence of COVID-19 in MM. Preventing community acquisition and deferral of non-emergent treatment of MM remain sensible measures. 
Author contributions $\mathrm{BD}$ conceived the project, collected the data, and wrote the manuscript. AD, SC, and P.H interpreted the data, commented, and edited the manuscript. All authors approved the final version of the manuscript.

\section{Compliance with ethical standards}

Conflict of interest The authors declare that they have no conflict of interest.

Publisher's note Springer Nature remains neutral with regard to jurisdictional claims in published maps and institutional affiliations.

\section{References}

1. He W, Chen L, Chen L, Yuan G, Fang Y, Chen W, et al. COVID-19 in persons with haematological cancers. Leukemia. 2020.
2. Liang W, Guan W, Chen R, Wang W, Li J, Xu K, et al. Cancer patients in SARS-CoV-2 infection: a nationwide analysis in China. Lancet Oncol 2020;21:335-7.

3. Yu J, Ouyang W, Chua MLK, Xie C, SARS-CoV-2 transmission in patients with cancer at a tertiary care hospital in Wuhan, China. JAMA Oncol. 2020. (in press)

4. Zhang L, Zhu F, Xie L, Wang C, Wang J, Chen R, et al. Clinical characteristics of COVID-19-infected cancer patients: a retrospective case study in three hospitals within Wuhan, China. Ann Oncol. 2020.

5. Sidaway P. COVID-19 and cancer: what we know so far. Nat Rev Clin Oncol. 2020.

6. Blimark C, Holmberg E, Mellqvist UH, Landgren O, Bjorkholm M, Hultcrantz M, et al. Multiple myeloma and infections: a populationbased study on 9253 multiple myeloma patients. Haematologica. 2015;100:107-13.

7. Zhang X, Song K, Tong F, Fei M, Guo H, Lu Z, et al. First case of COVID-19 in a patient with multiple myeloma successfully treated with tocilizumab. Blood Adv. 2020;4:1307-10. 